\title{
Anthropocene semiosis
}

\author{
Nigel Clark
}

Commentary on Ruddick, S M `Rethinking the subject, reimagining worlds’ Dialogues in Human Geography 7(2), 145-150.

\begin{abstract}
While affirming their more-than-human concerns, Ruddick (2015) proposes that there are limits to the capacity of relational ontologies to deal with the conflicting demands and extensive temporalities of the Anthropocene crisis. This commentary probes her alternative notions of a forcefulness and generativity that exceeds individuated bodies and of a semiosis that inheres in the workings of ecological and Earth systems.
\end{abstract}

\section{Keywords}

more-than-human, relational ontology, Anthropocene, Earth systems, semiosis

Building on a number of earlier excursions into the political ontologies of Spinoza and Deleuze (2008, 2010), Susan Ruddick's `Rethinking the subject, reimagining worlds’ (2015) both inherits and challenges the relational ontologies that have been gathering force in human geography and neighbouring disciplines over the last two decades or so. It might feel as though the west's centuries-old enthrallment to a human-nature divide has by now been well and truly displaced by a new set of ontological stories in which nature and society are understood to be mutually entangled and co-constitutive. But this is no time for theoretical complacency. While affirming the new ontological 'settlement' in many ways, Ruddick expresses doubts about the capacity of the relational makeover of the nature-society dichotomy to fully account for events and processes `whose temporalities extend millennia even aeons beyond our prior imaginings' (2015: 3). Taking the Anthropocene thesis as her incitement, she begins to lay out an alternative politics and ethics in which both force and signification are conceived as extending far beyond the bounds of any individuated being. 
There's a claim Ruddick makes about a tendency in relational ontological work to avail itself of the expanded sense of the subject offered by the embrace of the 'more-thanhuman' without making corresponding concessions or sacrifices (2015: 11). I suspect that this point has wider traction, not least in the reception of the Anthropocene thesis in certain strains of critical social thought. For many post-humanist scholars the scientific case for anthropogenic Earth system change is being taken as a vindication of the argument that no recourse can or ought to be made to a freestanding 'Nature' beyond the domain of human social existence. Quick to capitalise on the Anthropocene narrative of tightly coupled social systems and Earth systems, relational ontologists in particular now seem to be in a privileged position. Not only are they privy to a physico-material agency apparently disavowed in more discursive or linguistic styles of social thought, they also find themselves well placed to foreground the deep socialstructural divisions that are said to be passed over in more scientific constitutions of the Anthropos.

But what does it mean - as an ontological and political provocation - for humankind (or part thereof) to be construed as geological agents? Given that none of the social sciences have long-standing or even short-standing traditions for thinking deeply with or about geology, what exactly are our grounds for contributing usefully to debates over climate change, planetary boundaries or Earth system perturbations? Is it enough to identify the crucial social actors or isolate the social-structural determinants of 'anthroposcenic' agency and exposure? The achievements to date of relational ontologies clearly have some purchase on the mutual implication of social and physical processes evidenced in Earth systems science. But what do relational ontologists have to say about the abyssal reaches of geological time and dynamics- Ruddick's point above about aeon-spanning temporalities - without which the eye-blink of the current epoch makes little sense at all?

We can also play the geologic hand in the other direction. If the idea of humans 'becoming geologic' is challenging, what are social thinkers to make of earth processes that appear to be 'becoming human'? If the capacity of Earth systems to shift, recalibrate, reorder in response to human 'drivers' points to the messiness or mutability of the human-nonhuman interface, might this also have something say about the capabilities proper to the planet itself? Does the Earth need to 'become human' in order to express itself? Or is critical thought - even after all its linguistic turning - still 
too focused on sensations, meanings, negotiations in which it is presumed that we humans are the main event?

For me, it is in her manoeuvring between a world of much more-than-human forces and an Earth that is itself communicative that Ruddick most troubles and provokes existing Anthropocene discourse. While radical ecologists have long insisted that the living planet is meaningful in and of itself, and while natural scientists have compiled vast databases detailing the Earth system's recalibrations in response to internal and external forcings, neither offers an explicit articulation of a planet that characteristically communicates with itself - an astronomical body that is its own messaging system. It is this sense of the Anthropocene as semiosis in Ruddick's paper that want I focus on in what follows, after first looking more closely at her critical engagement with relational ontology.

Commenting a few years ago on Antonio Negri's engagement with Spinoza, Ruddick affirmed the broad intentions and spirit of the Italian political philosophers 'joyful' elaboration on the Spinozist notion of power, before adding: 'it is nonetheless frustrating in that it leaves unasked, and consequently unanswered, questions regarding difficult divisions, conflicts or divergent agendas in this process' (2010: 40). In the current paper we find a similar tone of affirmation coupled with frustration in regard to the claims of relational ontologies. While sharing the wish of such approaches to extend the scope of political and ethical relating beyond the bounds of our own species, Ruddick questions whether their privileging of attentive, caring, and convivial coexistence with nonhuman others can handle the surge of competing demands for attention and the extent or depth of strife sparked by the current planetary predicament (2015: 4-5).

In her view, one of the limits of much work in the relational ontological vein is that its attempts to stretch affective and generative 'becomings' beyond the bounds of the human have tended to hinge on encounters between members of our own species and other living beings who find themselves physically proximate to one another. While this displaces the idea of an autonomous `stand alone' human subject with more collaborative subjectivities, she argues, it still implies that one of the partners in the relationship is always 'us'. Moreover, despite - or by virtue of - a commitment to mutual transformation, the focus remains individuated entities (Ruddick, 2015: 2). In other words, for Ruddick, there is a tendency in relational ontologies to want to enrich 
enhance and extend processes of subject formation while holding on to the coherence and integrity of the organism. Thus `a humanist theory of the subject.... is never fully relinquished, but only extended to non-human others' (2015: 11).

We might anticipate that actor network theorists, among others, would retort that both their networks and their relata are always already constituted through transactions with multiple others. But it is notable that even in the offerings of ANT, it is rare to find sustained inquiry into situations that do not include humans as significant partners, just as it is rare across the broader sweep of relational ontologies to find deep engagement with forces, conjunctions and generative powers that operate beyond the bounds of living bodies. It is in this sense that Ruddick draws on Spinozist and Deleuzian notions of affect 'that downplay( ) the organism' (2015: 10) - an approach that resonates with recent work by Claire Colebrook (2010: 77). If Spinoza provides a vision of composite bodies whose form and potentiality emerge out of conjoining of powers or forces, Ruddick takes from Deleuze an extrapolation of this becoming with and through heterogeneous bodies beyond the human domain.

But the importance of creative forces that precede and exceed the organic body is only half of the story. What also interests Ruddick are the ways in which force itself might be imbued with capacities most often confined by critical thinkers to our own species (or at best the uppermost branches of the animal kingdom). It is in this regard that she turns to the Charles Sanders Pierce's positing of more-than-human semiosis (2015: 1318). What Ruddick finds in the work of the pragmatist philosopher is the pioneering proposition that constitutive exchanges between bodies entail relationships that are as much about signification as they are about force. Pierce, she insists, does the groundwork for a theory of sign-play or communication that extends far beyond interhuman exchanges to take in transactions between other life forms and even amongst the non-living: 'In the Peircean formulation, meaning-factors are at work across the entire universe of things' (Ruddick, 2015: 15). In this sense, for Ruddick, becomings that are too often reduced to relationships between organisms and their environment can be reconstrued not only in terms of more dispersed activations of matter-energy, but as semiotic negotiations. 'Here, over a long time frame,' she writes, 'the complex adjustments between organic and inorganic matter might be considered a kind of communication, imbued with meaning factors' (2015:15). 
It is this thinking beyond the organism in terms of both force and semiosis that for me makes for such an intriguing and original conceptualisation of the Anthropocene. For Ruddick, the predicament of the Anthropocene is not only one of human 'forcings' impacting on the dynamics of the ecological and Earth systems. It is also about the interruption or over-coding of established pathways of communication: the scrambling of a whole range of geoclimatic, chemical or biotic messages that orchestrate the hatching, mating, feeding, resting or migrating patterns of living beings. As Ruddick explains:

Disruption of visual, auditory, olfactory, electromagnetic and other cues in the landscape influence the behaviors of animals and organisms in broadly defined geo-systems: our knowledge of perceptual cues (or meaning factors) is increasingly important as logging, mining, urban and other developments alter textual cues of the landscape (2015:18).

In Ruddick's broader picture, it would appear, it is this planet-wide meshwork of messaging - as much as any material-energetic forcefulness - that grants the Earth its systemicity, its dynamics, its state-shifting capacities. Critical social analysis of climatology and Earth system science has often evinced a deep suspicion of efforts to model earth processes in their totality - on account of the way that technological prostheses enable an imagining of the Earth from an abstracted, placeless 'beyond'. Part of the appeal to me of Ruddick's notion of an auto-communicative planetarity is that it unsettles the idea that human-driven informationalization of the Earth is a dangerous supplement to the planet's own groundedness and self-consistency. In its place, we are offered an Earth that is always already communicating with itself: transmitting, receiving, calculating, auto-affecting. After all, it might be added, modelling the impact of increasing greenhouse gas emission on global climate only makes sense on the back of an understanding that the Earth system is constantly recalibrating itself with every new emission.

In this way, Ruddick's material-semiotic vision of the planet resonates with some rare extrapolations of Jacques Derrida's deconstructive logic into the realms of the geophysical. Vicki Kirby $(1997,2011)$, in particular, has long insisted that the troubling of the duality of force and signification was always intended by Derrida to be extended far beyond the domain of human communication (and in this regard its worth recalling that an affirmative reading of Pierce is shared reference point in the work of 
Deleuze and Derrida). For Kirby, it is not only that the 'telling flesh' of living organisms that is imbued with messaging capabilities, but that the Earth and cosmos ought to be seen as compendia of communicative capacities. As well as evoking the 'spooky action at a distance' that physicists have discerned at the quantum scale (Einstein cited in Kirby 2011: 126), she explores lightning's uncanny 'awareness’ of topographic high points in its passage from sky to earth - and draws our attention to way that the overall balance of positive/negative electrical valences of the Earth's entire atmosphere is recomputed with every single lightning strike (2011: 10-12). In this regard, Kirby proposes that 'what we conventionally call Nature is as actively literate, numerate, and inventive as anything we might include within Culture' (2011: 66). And she goes on to wonder whether such self-sensing could be considered 'as the Earth's own scientific investigations of itself' (2011:34).

Such incitements to think force and signification together also have repercussions for the way we think about the origins of the ecological and Earth systemic ruptures currently being shorthanded by the Anthropocene concept. Whereas scientific causal accounts have generally fallen back on quite conventional notions of socio-technical turning points and linear growth curves, and while critical social science originary stories seem oddly reluctant to stray far from received social-structural or 'political' narratives, an Earth imagined as always already computing, conversing and negotiating with itself raises some provocative possibilities. The claim that our own species - or at least significant fractions of the human collective - are capable of interrupting or overwriting the messaging capabilities of biotic and Earth systems raises questions about the source of such communicative scrambling. Is it only our own species that messes with planetary signifying processes? Or are Earth systems - or the various components and sub-divisions of the planet - themselves capable of shortfalls in communication, of misinterpreting or over-reacting to signals? (cf Ruddick, 2015: 17).

This might be one reading, for example, of the Derridean structural logic of the trace which proposes that any entity that harbours non-identity within itself has the capacity to turn against itself (Derrida, 1981: 119). In his later work, Derrida developed this train of analysis into the notion of autoimmunization: the idea that any system or being which has the ability to modulate the flows between its own interior and the world beyond also contains the possibility of misreading vital signs. Or in his own words: '... this strange illogical logic by which a living being can spontaneously destroy, in an autonomous fashion, the very thing within it that is supposed to protect it against the 
other, to immunize it against the aggressive intrusion of the other' (Derrida: 2005: 123). Although not pursuing the idea, Derrida himself suggested that this dynamic might be extended to 'the fragile destiny of the planet' (2005: 117; see also Clark, 2012: 273).

However far we chose to push such provocations, what Ruddick shares with both Kirby and Colebrook is a sense that one of the problems with recent relational ontologies is that they have tended to conceive of themselves - usually in explicit contradistinction a perceived prevalence of more discursive, cultural or linguistic approaches - as materialisms. And in this way, they foreclose on important theoretical resources for deconstructing or otherwise negotiating the matter-ideality divide - a duality that may well be more deeply-engrained and more pressing than the humannonhuman rift. As Elizabeth Grosz has recently added, reviewing a cast of philosophical figures that includes Spinoza, Deleuze and Pierce's compatriot pragmatist William James: 'each of these philosophers, while appearing to be materialists, and addressing questions about the world through materialism, remain attached to a concept of the ideal, ideality, or conceptuality that is irreducible to anything material' (2014: unpag.).

Following Ruddick's reading, then, the imputing of properties of communicability to the geophysical Earth might help free certain relational ontologies from their vitalist enthralment with the organism - thus relieving entities, fields or systems of the need to be human-like or even life-like in order to be articulate. At the same time, working the semiotic-material conjuncture from the other direction suggests a geology or a minerality that is proper to the living being (see Clark, 2012). It raises the possibility that 'life itself' might be imbued with an essential - though not necessary stable materiality: the kind of base matter that is often passed over in post humanist thought in favour of affirming the endless malleability of biological becoming. In this sense, the 'becoming geologic' of human actors implied in the Anthropocene thesis might gesture not only 'outward' toward the forcing of Earth systems by the powers of social agency, but also draw our attention to the potentiality of the earthy matter enfolded within the collective and individuated bodies of our species. As Kathryn Yusoff puts it, it points towards a geology that is no longer 'bracketed out from subjective experience or placed outside the corporeal body' (2015: 6). 
If there is at once a human geology - an elementality with its own forceful and generative logic at work within our very being - and a semiosis with its own complex and perhaps contrary logic at work all the way up to the planetary scale -we can begin to see why critical social thought ought to resist scoring easy points off the Anthropocene. No less than natural scientists or more cultural and linguistically oriented social scientists, social thinkers of a relational ontological leaning still have a lot to learn. By the same token, we can start to see the sheer scale and perplexity of the ethical-political challenge Ruddick sets herself. She is keen that extending the scope of politics and ethics far beyond the human and the organismic does not result in dissipation into ineffectuality (2015: 2), to ensure that - to borrow a formulation from Hayden Lorimer - 'what emerges is not simply a smear of equivalence' (Lorimer, 2005: 88). She is likewise attentive to the inverse possibility, discernable in much stridently radical or progressive engagement, of an investment in power imbalances seen to be so deeply incised as to appear insurmountable (Ruddick, 2008: 2600). Ruddick's alternative, elaborating on Spinoza's notion of potentia, involves making informed, inspired but tough choices about what nonhuman forces we ally ourselves with so as best to power or energise our contests with the all-too-human entrenchments of political power (2015: 18-19).

This is clearly a project without templates or guarantees. The drive to promote collaborations that enhance our capacities and to resist entanglements that sap our energies comes with no dependable guidebook. For in a volatile world what may be empowering in one moment or context may be a hindrance in another. Ruddick's ambitious manoeuvring between force and signification does not make these decisions any easier, especially if we also seek to account for the indeterminacy that characterises any signifying process: opportunities for misreading, slippery translation and overreaction that may inhere not only in human sociality but in the Earth's own interactions with itself. It would seem that the best we can hope for is interminable experimentation - trials that are inextricably material and communicative. As Ruddick has earlier observed 'For Deleuze the genesis of thought is not a model of the same, but a co-creation of something unforeseen, a 'long-lasting affair with experimentation"' (2010: 36). Much the same would seem to apply to the political and ethical possibilities sketched out in the current paper.

More surely, what Ruddick helps us to see is that Anthropocene is not simply a problem any of us can solve. If human agency is implicated in and subtended by 
earthly dynamics whose force and significance stretches across geological time, the predicament signposted by the Anthropocene is not simply ours to comprehend, undo or recompose. While it is important that critical social thinkers keep reminding us of the inequalities and divisions that sunder the Anthropos, it is just as crucial that we consider the contribution of turbulent and mutable Earth processes to the differences within our species being. And indeed, if we follow Ruddick's suggestion that this is a planet that queries, computes and converses, we might even wonder whether such a differentiated humanity is itself one possible answer to conundrums posed by a likewise divided and non-self-identical Earth.

\section{References}

Clark N (2012) Rock, life, fire: Speculative geophysics and the Anthropocene. Oxford Literary Review. 34(2): 259-276

Clark N (2012) Sex, politics and inhuman artistry. Dialogues in Human Geography 2(3) 271-275

Colebrook C (2010) Deleuze and the Meaning of Life. London, UK and New York, NY: Continuum International Publishing Group.

Derrida J (1981) Dissemination. Chicago: University of Chicago Press.

Derrida J (2005) Rogues: Two Essays on Reason. Stanford, CA: Stanford University Press.

Grosz E (2014) Ontogenesis and the ethics of becoming, Interview with Society and Space Open Site http://societyandspace.com/material/interviews/ontogenesis-and-theethics-of-becoming-an-interview-with-elizabeth-grosz-by-kathryn-yusoff/ (accessed 19 July 2015)

Kirby V (1997) Telling Flesh: The Substance of the Corporeal. London: Routledge

Kirby V (2011) Quantum Anthropologies: Life at Large. Duke University Press: 
Durham, NC and London, UK.

Lorimer H (2005) Cultural geography: the busyness of being 'more-thanrepresentational. Progress in Human Geography 29(1): 83-94

Ruddick S (2008) Towards a dialectics of the positive. Environment and Planning A. 40: $2588-2602$.

Ruddick S (2010) The Politics of affect: Spinoza in the work of Negri and Deleuze, Theory, Culture \& Society 27: 21-45.

Ruddick S (2015 forthcoming) Rethinking the subject, reimagining worlds.

Dialogues in Human Geography.

Yusoff K (2015 forthcoming) Anthropogenesis: Origins and endings in the Anthropocene Theory, Culture \& Society

Corresponding author:

Nigel Clark, Lancaster Environment Centre, Lancaster University, Lancaster LA1 4YW, UK.

Email: n.clark2@lancaster.ac.uk 